\title{
Cross-layer design using multi-channel system in WiMAX mesh networks
}

\begin{abstract}
The WiMAX mesh networks based on IEEE 802.16 standard is a key topology for the next generation wireless networking. However, interferences from transmission of the neighboring nodes within the mesh networks are inevitable. Cross-layer design for tree-type routing and level-based centralized scheduling is proposed as the optimization strategy. The cross-layer design here is relying on the routing information in network layer and centralized scheduling in the medium access control (MAC) layer. This paper proposes a centralized scheduling algorithm that can reduce interferences by constructing routing tree with multi-channel single transceiver system in WiMAX mesh networks. In this algorithm, each node has one transceiver that can be tuned to any of the channels, intending to eliminate the secondary interference. The results of analysis show that this proposed algorithm greatly improves the length of scheduling, channel utilization ratio (CUR) and throughput.
\end{abstract}

Keyword: 802.16d; Routing tree; Scheduling; Multi-channel 